\title{
Influence of imaging ability on word transformation
}

\author{
PHILIP A. ALLEN, BENJAMIN WALLACE, and FRANK LOSCHIAVO \\ Cleveland State University, Cleveland, Ohio
}

\begin{abstract}
In the present study, we examined whether individual differences in imaging ability affect visual word recognition. Poor and vivid imagers performed a naming task that involved nonreversed (e.g., JUMP) and reversed (e.g., PMUJ) words (Experiment 1). Poor and vivid imagers were also tested on a naming task that was controlled for verbal ability; all the words were reversed and presentation time was varied (Experiment 2). In both experiments, imaging ability interacted with task difficulty, suggesting that individual differences in imaging ability affect visual word recognition. Specifically, the present data suggest that poor imagers may be less efficient than vivid imagers at processing words analytically. The data are interpreted within a limited-capacity, hybrid, word recognition model, in which words can be processed as either word-level or letter-level codes.
\end{abstract}

It has been demonstrated in a number of studies that subjects' imaging ability affects perceptual and cognitive performance. For example, Wallace (1988) had subjects participate in a visual search task in which they were asked to find embedded objects within pictorial scenes. In general, vivid imagers (as judged by performance on the Vividness of Visual Imagery Questionnaire, or VVIQ; Marks, 1973) performed the task better than poor imagers.

Vivid imagers also perform better on a gestalt closure task. Wallace (1990) required subjects to identify fragmented stimuli in the Closure Speed Test (Thurstone \& Jeffrey, 1966) and in the Street Test (Street, 1931). The greatest number of correct closures was reported by vivid imagers. Also, Wallace (1991b) examined proofreading accuracy as a function of imaging ability. Six one-page passages from various texts were prepared in such a way that each contained 26 misspelled words. A misspelled word was produced by substituting a middleposition letter with another letter of either similar or dissimilar shape. The subjects who were judged to be vivid imagers made fewer proofreading errors than did the poor imagers. This effect was qualified by an interaction between imaging ability and letter similarity. When the substitute letter had a shape that was similar to the letter it replaced, the poor imagers made more errors than did the vivid imagers. However, when the substitute letter was dissimilar to the letter it replaced, there were no

This research was supported by National Institutes of Health Research Grant AG09282 to the first author. We thank Geoffrey Loftus, Lester Krueger, Peter Dixon, and one anonymous reviewer for comments on an earlier version of the manuscript. Also, we thank Tim Weber and Therese Finnan for technical assistance. Address correspondence to P. Allen, Department of Psychology, Cleveland State University, Euclid Ave. at East 24th Street, Cleveland, OH 44115 (e-mail: p.allen@csuohio.edu) differences in proofreading accuracy across imaging ability.

In essence, these studies have shown that vivid imagers are more adept in performing a variety of tasks. This may be partly because poor imagers appear to process information in an analytic style and not a holistic style (Crawford \& Allen, 1983). Such analytic processing generally produces slower and less efficient responding (see Wallace, 1991a). For example, in Wallace's (1991b) study, when the subjects proofread the text, the poor imagers processed the text by reading it from left to right while searching for misspelled/mistyped words. That is, they appeared to be searching for errors by examining the letters of each word one at a time. On the other hand, the vivid imagers seemed to search in a holistic fashion, by examining the words of an entire line of text at a time.

However, there are instances in which poor imagers appear to use holistic rather than analytic processing strategies for identifying words. Allen, Wallace, and Waag (1991) examined letter identification performance on a task in which subjects matched a target letter to the initial letter of a subsequently presented probe word. The poor imagers showed a word frequency advantage (across four word frequency categories) for the letter identification task, which suggests that these subjects used word-level (holistic) codes to indirectly access letter-level (analytic) information (Allen, Madden, \& Crozier, 1991; Allen, Wallace, \& Waag, 1991). The vivid imagers, on the other hand, showed a nonmonotonic RT effect across word frequency, indicating that they processed letter strings as words as well as individual letters.

This interpretation is based upon the parallel input serial analysis (PISA) model, developed by Allen and Madden (1990), and Allen and Emerson (1991), who argued that a nonmonotonic function for reaction time (RT) across word frequency for a precued, letter identi- 
fication task indicates that separate word-level and letter-level input modules are involved in a processing "horse race" to a central processor. Note that in the present application, holistic processing refers to representing an entire word as a single unit (similar to a global "picture"), whereas analytic processing refers to representing a word as individual letters. In this view, the word-level module encodes words on the basis of the spatial-frequency pattern of the entire word (e.g., JUMP), whereas the level-level module encodes words on the basis of the spatial-frequency pattern of individual letters (e.g., J U M P) (Allen \& Emerson, 1991). The central processor automatically selects the output of the winning input code, even if the information from the winning input code is not appropriate for the task at hand (if the winning code cannot be used to solve the task at hand, the central processor switches attention to another input channel; see Fodor, 1983). Word-level codes are assumed to be output progressively more rapidly as word frequency increases, but letter-level codes are unaffected by word frequency (Johnson, Allen, \& Strand, 1989). Within the framework of hybrid, holistically biased models of word recognition, such as the PISA model (see, e.g., Allen \& Emerson, 1991; Allen \& Madden, 1990; Johnson et al., 1989; see also Healy, Conboy, \& Drewnowski, 1987; Healy, Oliver, \& McNamara, 1987; Krueger, 1989), a nonmonotonic RT function across word frequency suggests that both word-level and letter-level codes are processed in parallel. Figure 1 illustrates the processing dynamics of the PISA model across four different word frequency categories for letter identification.

However, as noted above, Allen, Wallace, and Waag (1991) found that poor imagers showed a fairly monotonic RT word frequency advantage for the same precued, letter identification task for which typical young adults and vivid imagers showed the aforementioned nonmonotonic function. It was concluded that the poor imagers identified words by using the word-level channel (i.e., the word frequency advantage indicated that words were accessed in the mental lexicon) and then used top-down processing to determine the component letters of a word (Allen \& Emerson, 1991; Allen, Wallace, \& Waag, 1991). The data were consistent with an interpretation of individual differences in processing resources. ${ }^{1}$ That is, it takes more processing resources to represent a six-letter word as six separate codes (i.e., a letter-level, or analytic, code of a six-letter word) than to represent that same word as a single code (i.e., a wordlevel, or holistic, code of a six-letter word) (Allen \& Emerson, 1991). If one assumes that poor imagers possess fewer processing resources than vivid imagers, it is reasonable to assume that poor imagers will be forced to use a processing method that minimizes processing resources. Hence, Allen, Wallace, and Waag suggested that poor imagers use a holistic strategy to complete a letter identification task, whereas vivid imagers use both holistic and analytic strategies. These data imply that task demands and individual differences affect the type of processing that poor imagers will use. Therefore, even though vivid imagers typically use a holistic processing style and poor imagers use an analytic processing style (e.g., Crawford \& Allen, 1983), the processing-resource demands of a task may prevent subjects from using these established styles.

To test this hypothesis, we needed a task in which subjects would be forced to use an analytic word identification strategy. One such task is the mental transformation of a word. For example, if subjects are presented with a four-letter string in a reversed order (e.g., PMUJ), as contrasted with the same string in the correct order (e.g., JUMP), then processing differences between poor and vivid imagers should become apparent if poor imagers possess fewer processing resources. This is be-

$\begin{array}{lc}\begin{array}{l}\text { Very-High } \\ \text { Medium-High }\end{array} & \text { LLLLL } \\ \text { Wow } & \text { WWWWLLLLL } \\ \text { LerY-Low } & \text { LLLLL } \\ \text { Early } & \text { WWWWW } \\ \end{array}$

Figure 1. Time course of availability for letter-level (L) and word-level (W) codes for words with very high, medium high, low, and very low frequencies. The number of consecutive adjacent letters represents the time it takes the central processor to decode a word-level or letter-level code. For example, after the fifth " $W$ " the word-level code has been output from the input module, and the central processor has decoded this output. According to the PISA model (Allen \& Madden, 1990), the time course for letter-level output is the same for all word frequency categories. However, word-level codes are assumed to be output progressively faster as word frequency increases. Hence, the PISA model predicts that letter identification reaction time in words with medium-high frequencies should take longer than letter identification in other frequency categories, because the decoding of the word-level code for words with medium-high frequencies delays access to the letterlevel code needed to complete this task relative to letter-level access time for other word frequency categories. However, for lexical decision and pronunciation tasks (in which the word-level rather than letter-level code is used to complete the task at hand), the PISA model predicts a word frequency disadvantage. 
cause reversed words require an analytic process (which requires a relatively large amount of processing resources), whereas nonreversed words tend to involve holistic processing (e.g., Allen, Wallace, \& Weber, in press; Koriat \& Norman, 1985, 1989), which requires fewer processing resources (e.g., Allen et al., in press). That is, it is impossible to rotate a reversed word as a single unit around a central axis (as would be necessary for holistic processing) and have the resulting stimulus be a real word. If a reversed word were to be rotated, it would produce a mirror-image stimulus that would not result in an identifiable word (e.g., if an individual actually rotated PMUJ around a central axis, then tumq would result instead of JUMP). Thus, subjects would be forced to reorder the reversed letters in an analytic, letter-by-letter process in order to recognize a reversed word.

The present mental transformation task consisted of a naming task. There is evidence that the same basic PISA architecture, using input modules and a central processor with a switching mechanism that was originally applied to letter identification (e.g., Allen \& Emerson, 1991; Allen \& Madden, 1990), can also be used to account for lexical decision and naming tasks (Allen, McNeal, \& Kvak, 1992; Allen et al., in press). The key difference is that, in a naming task with untransformed stimuli, the word-level code provides the pertinent information, whereas in a letter identification task, the letter-level code is pertinent. When a nonreversed word is presented on a naming task, subjects typically use the unitary, holistic code of the word to recognize it (Allen \& Emerson, 1991). However, when a word is reversed, as was done in the present experiments, the PISA model predicts that individuals will have to (1) mentally reverse the letters so that a word is formed, and (2) "superpose" the letter-level code of the word into a "pseudo-wordlevel" code, so that the mental lexicon can be accessed (Allen \& Emerson, 1991; see also Figure 2). This superposition process is processing-resource intensive and time consuming, because it is performed by the relatively slow (but "smart") central processor rather than by the fast (but "dumb") input processors (Allen \& Emerson, 1991). Note that for nonreversed and reversed words, the PISA model predicts a monotonic word frequency advantage. The advantage of the present task is that it allows an examination of primarily holistic processing for nonreversed words, but allows an examination of analytic processing for reversed words.

There were two main goals in the present investigation. First, we wanted to test whether subjects typically use holistic processing in order to recognize familiar patterns, but use analytic processing in order to recognize unfamiliar patterns (Allen \& Emerson, 1991; Koriat \& Norman, 1985, 1989). In Experiment 1, we tested this hypothesis by using a naming task in which half of the words were presented in a nonreversed manner (e.g., JUMP), while the other half were presented in a reversed manner (e.g., PMUJ). As noted previously, a mental rotation of an integral whole around a central axis cannot

\section{TASK \\ UTILIZATION}

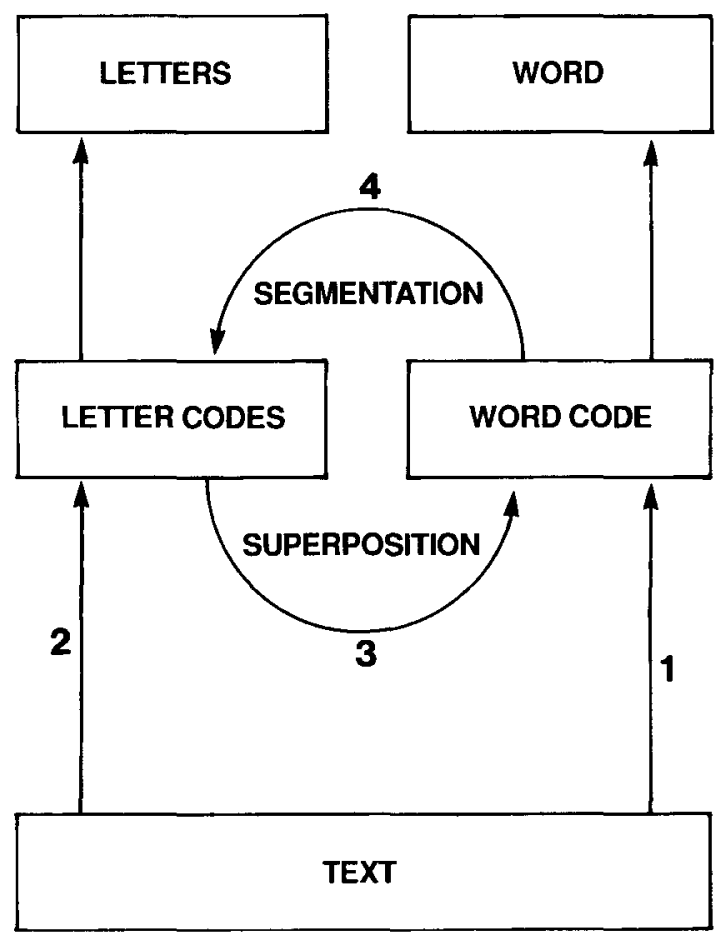

Figure 2. There are four pathways in the hasic PISA model (from Allen, Wallace, \& Weber, in press). Two pathways (labeled 1 and 3) form word-level codes. Pathway 1 is a direct route, where word-level codes are formed from the spatial-frequency pattern of an entire word. Pathway 3 is an indirect route that is used to form word-level codes when they are necessary to complete the task at hand, but when the spatial-frequency pattern of the word is unfamiliar, and when the spatial-frequency pattern of component letters is familiar ( $\mathrm{e} . \mathrm{g}$, when a word is reversed or presented in mixed case). In this route, the central processor superposes the letter-level code into a pseudoword-level code. However, this route is time consuming and processing-resource consuming. Pathway 2 is a direct route for letter-level codes, and Pathway 4 is an indirect route for the formation of letterlevel codes (i.e., segmentation) that is analogous to Pathway 3 for words.

form a word on reversed trials. Thus, the subjects could not use holistic processes to reverse and recognize these words. If subjects tend to use holistic word recognition processes when word shape is adequately familiar, but tend to use analytic word recognition processes when letter shape, but not word shape, is adequately familiar, then Experiment 1 should reveal faster pronunciation times for nonreversed words than for reversed words. Also, word length should have a greater effect on reversed words than on nonreversed words, because holistic processing should be less affected by word length (Koriat \& Norman, 1985, 1989). This is because holistic processing treats a word as a single unit, but analytic processing represents the letters of a word separately; therefore, increasing the number of letters increases the 
number of codes (Allen \& Emerson, 1991; Koriat \& Norman, 1985).

The second goal of the present study was to determine whether individual differences in imaging ability affect analytic word processing resources. That is, Allen, Wallace, and Waag (1991) found evidence that poor imagers had fewer analytic processing resources than vivid imagers. However, the present experiments allowed a more direct test of this hypothesis, because the subjects were forced to use analytic processing for reversed words. It was predicted that word length and transformation type in Experiment 1 would interact with imaging ability. This is because the differences in processing resources between poor and vivid imagers (Allen, Wallace, \& Waag, 1991) should especially affect letter-level (analytic) processing, for which increased word length and reversed words are predicted to affect performance. In Experiment 2 (a naming task, in which words were reversed and display time was manipulated), it was predicted that, if vivid imagers evidence more analytic processing resources than poor imagers, imaging ability would interact with word length and presentation time even when verbal ability was equated across imaging type.

\section{EXPERIMENT 1}

In the first experiment, we tested poor and vivid imagers (as determined by the Test of Visual Imagery Control, or TVIC; Gordon, 1949) on a pronunciation task. Half of the trials involved reversed words (e.g., PMUJ) and half involved nonreversed words (e.g., JUMP). The subjects were instructed to mentally transform the reversed words (e.g., PMUJ) into their correctly spelled form (i.e., JUMP) and then pronounce the correctly spelled words. No mental transformation was necessary for nonreversed words. This experiment was conducted in order to determine the influence of processing type (holistic vs. analytic) and imaging ability on performance.

\section{Method}

Subjects. A total of 40 undergraduate subjects from Cleveland State University participated in this experiment for credit in an in. troductory psychology course. All of the subjects reported normal or corrected-to-normal vision and were able to accurately recognize words that were presented on a computer screen prior to the actual experiment. The TVIC was used here because it has been shown to be a reliable measure of the ability to manipulate an image (see Chara, 1989; Wallace, 1991a) and does not simply measure imaging ability, as is the case with the VVIQ. The 20 subjects who responded "yes" to at least 10 items on the 11 -item TVIC (top 25 th percentile) were designated vivid imagers, and those who responded "yes" to 6 or fewer items on the TVIC (bottom 25 th percentile) were designated poor imagers.

Apparatus. All the subjects were individually tested on an IBM PS/2, Model 30 (286) microcomputer with a VGA card and a Mitsubishi Diamond Scan monitor. All the stimuli were presented in white on a black background. The subjects' responses were collected by using the Micro Experimental Laboratory (MEL) software (Schneider, 1988). Naming responses were collected through a Radio Shack microphone and the voice-activated response key of the MEL response box. Only correct RTs were analyzed, and inadvertent responses were not entered into the analyses.

Materials. The stimuli were grouped into four word frequency categories (see Kučera \& Francis, 1967): very low frequency (VLF; range $=1-5$ occurrences in the Kučera \& Francis corpus of slightly over one million words, $M=2.5$ ), low frequency (LF; range $=40-54$ occurrences, $M=48$ ), medium high frequency (MHF; range $=155-235$ occurrences, $M=193$ ), and very high frequency (VHF; range $=240-660$ occurrences, $M=364$ ). This stimulus set was based on the one used by Allen and Emerson (1991, Experiment 1) and on those used by Allen et al. (1992), which showed monotonic word frequency advantages for a lexical decision task.

In the present naming task, each letter was $0.28^{\circ}$ wide and $0.56^{\circ}$ high, with a $0.03^{\circ}$ separation between letters. An entire six-letter word was $1.85^{\circ}$ wide. The viewing distance for all the subjects was approximately $50 \mathrm{~cm}$, and the approximate display luminance was $25 \mathrm{~cd} / \mathrm{m}^{2}$.

Procedure. Each subject was individually tested with a naming task. On each trial, the participants were instructed to pronounce a single four-, five-, or six-letter word that was presented in the center of the display monitor. If a subject did not respond within $10 \mathrm{sec}$ after a target word was presented, the program continued on to the next trial (i.e., these trials were treated as errors, but they were not used in the RT analysis). Twenty practice words and 216 experimental words were presented to each subject. The experimental words were randomly selected, without replacement by the computer program, from the analogous cells of the 432 word stimulus set used by Allen et al. (1992). There was a $500-\mathrm{msec}$ delay between trials. All the words were presented in lowercase letters to better approximate the typical reading environment. Each subject's RT was measured from the onset of the word stimulus to the time when a subject's voice activated the response key. After each response, the reversed or nonreversed target word was followed by a fixation point (a "+") for $500 \mathrm{msec}$ before the next word was presented.

There were 108 nonreversed words (e.g., JUMP) and 108 reversed words (e.g., PMUJ), which were randomly presented within a single molar block of trials. None of the reversed stimuli formed another word in their reversed form (e.g., TAR to RAT). Within each transformation type (nonreversed vs. reversed), word length (four-, five-, and six-letter words) and word frequency (VHF, MHF, LF, and VLF) were also manipulated. Thus, each transformation type $\times$ word length $\times$ word frequency cell contained nine trials per subject.

\section{Results}

Latency data. The RT data from Experiment 1 (see Figure 3 ) were analyzed with a 2 (imaging ability: vivid vs. poor imagers) $\times 2$ (transformation type) $\times 4$ (word frequency) $\times 3$ (word length) mixed-design analysis of variance (ANOVA; $2 \times 2 \times 4 \times 3 \times 9=216$ experimental trials). The imaging-ability variable was a between-subjects factor, but the remaining three independent variables were within-subjects factors. The vivid imagers responded more rapidly $(1,237 \mathrm{msec})$ than the poor imagers $(1,384 \mathrm{msec})\left[F(1,38)=5.67, M S_{\mathrm{e}}=\right.$ $913,337, p<.05]$, nonreversed words were responded to more rapidly than reversed words (789 msec vs. $1,832 \mathrm{msec})\left[F(1,38)=480.33, M S_{\mathrm{e}}=543,963, p<\right.$ $.001]$, and the poor imagers showed longer mental transformation latencies (reversed - nonreversed = $1,956 \mathrm{msec}-812 \mathrm{msec}=1,144 \mathrm{msec}$ ) than did the vivid 

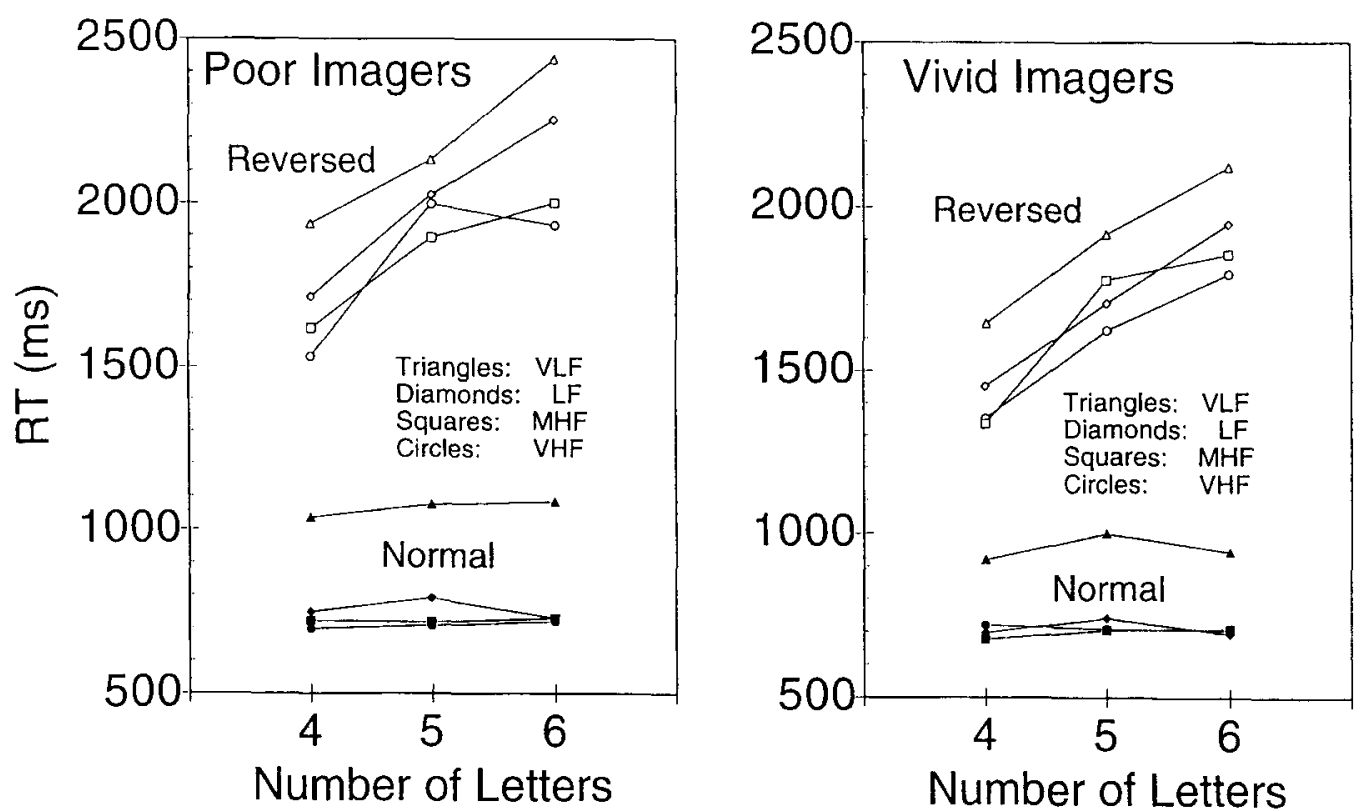

Figure 3. Vivid and poor imagers' reaction time (RT) data for Experiment 1 are plotted as a function of transformation type (reversed vs. normal), word length $(4,5$, or 6 letters), and word frequency (very low frequency, VLF; low frequency, LF; medium high frequency, MHF; very high frequency, VHF).

imagers $(1,708 \mathrm{msec}-766 \mathrm{msec}=942 \mathrm{msec})$ $\left[F(1,38)=4.48, M S_{\mathrm{e}}=543,963, p<.05\right]$ (see Figure 3 ).

There was also a reliable word frequency advantage $(\mathrm{VHF}=1,206 \mathrm{msec}, \mathrm{MHF}=1,227 \mathrm{msec}, \mathrm{LF}=$ $1,290 \mathrm{msec}, \mathrm{VLF}=1,519 \mathrm{msec})[F(3,114)=74.00$, $\left.M S_{\mathrm{e}}=67,023, p<.001\right]$, and a word-length disadvantage (four letters $=1,174 \mathrm{msec}$, five letters $=1,345 \mathrm{msec}$, six letters $=1,414 \mathrm{msec})\left[F(2,76)=61.53, M S_{\mathrm{e}}=\right.$ $79,197, p<.001]$. Transformation type interacted with word length $\left[F(2,76)=68.25, M S_{\mathrm{e}}=62,844, p<\right.$ $.001]$, and transformation type interacted with word frequency $\left[F(3,114)=3.35, M S_{\mathrm{e}}=48,325, p<.05\right]$. The former interaction resulted from a greater word-length disadvantage for reversed words (six letters $=2,042 \mathrm{msec}$ - four letters $=1,572 \mathrm{msec}=470 \mathrm{msec}$ ) than for nonreversed words $(786 \mathrm{msec}-776 \mathrm{msec}=10 \mathrm{msec}$ ), and the latter interaction resulted from an increase in RT across word frequency that was less gradual for nonreversed words than for reversed words (see Figure 3). However, when the data were analyzed separately by transformation type, nonreversed $[F(3,114)=124.74$, $\left.M S_{\mathrm{e}}=20,658, p<.001\right]$ and reversed $[F(3,114)=$ $\left.26.88, M S_{\mathrm{e}}=94,690, p<.001\right]$ words both revealed reliable word frequency effects. Finally, when the data were analyzed separately by imagery ability, the main effects for transformation type, word frequency, and word length, as well as the transformation type $\times$ word length interaction remained reliable for the vivid and poor imagers.

The critical result from the Experiment 1 RT data was the imaging type $x$ transformation type interaction. To further interpret this interaction, we examined the simple main effects of imaging ability at both levels of transformation type (i.e., reversed vs. nonreversed). For nonreversed trials, there was no main effect for imaging type $\left[F(1,38)=1.98, M S_{\mathrm{e}}=10,721\right.$, $p>$.15]. However, for reversed trials, the poor imagers did take significantly longer to pronounce a word than the vivid imagers did $\left[F(1,38)=5.54, M S_{\mathrm{e}}=110,720\right.$, $p<.05]$.

Error data. The error data for Experiment 1 are presented in Figure 4. The ANOVA conducted on these data indicated that the subjects were less accurate on reversed trials (mean error $=28.2 \%$ ) than on nonreversed trials $(4.0 \%)\left[F(1,38)=160.33, M S_{\mathrm{e}}=7.11, p<.001\right]$, that errors increased as word frequency decreased (VHF = $13.1 \%, \mathrm{MHF}=13.9 \%, \mathrm{LF}=14.5 \%, \mathrm{VLF}=23.1 \%$ ) $\left[F(3,114)=37.81, M S_{\mathrm{e}}=1.12, p<.001\right]$, and that errors increased with word length (four letters $=8.02 \%$, five letters $=16.4 \%$, six letters $=21.0 \%)[F(2,76)=$ $\left.39.88, M S_{\mathrm{e}}=1.66, p<.001\right]$. A transformation type $\times$ word length interaction $\left[F(2,76)=40.23, M S_{\mathrm{e}}=1.46\right.$, $p<.001]$ indicated that the word-length effect for reversed words was relatively greater than that for nonreversed words (see Figure 4). Finally, a transformation type $\times$ word frequency interaction $[F(3,114)=7.76$, $\left.M S_{\mathrm{e}}=0.84, p<.001\right]$ revealed that there was a greater word frequency effect for reversed words than for nonreversed words (see Figure 4). There was no main effect for imaging ability $(F<1.0)$.

As was the case for RTs, when the error data were analyzed separately by imaging ability (i.e., vivid vs. poor), both of the groups showed main effects for transformation type, word frequency, and word length, as 

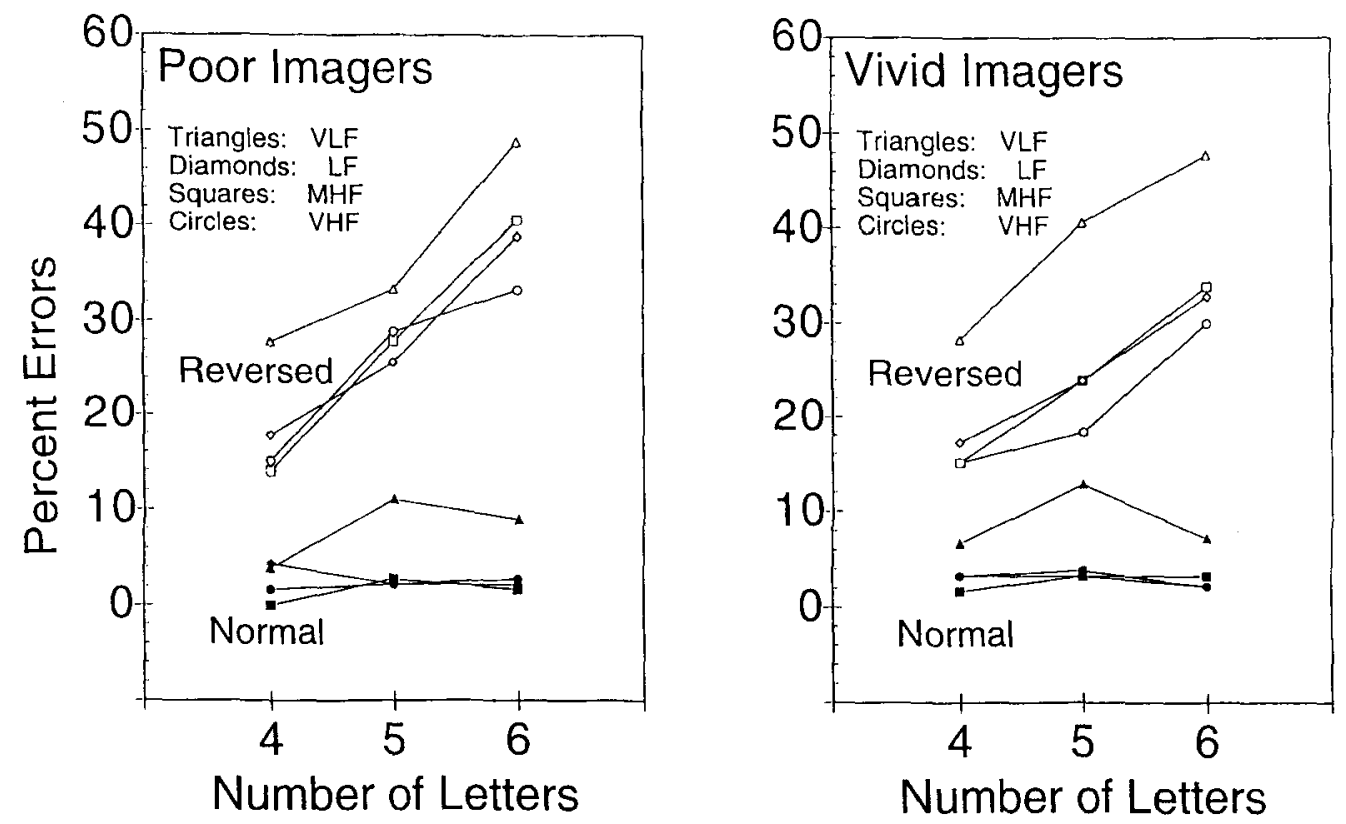

Figure 4. Vivid and poor imagers' mean percent error data for Experiment 1 are plotted as a function of transformation type (reversed vs. normal), word length $(4,5$, or 6 letters), and word frequency (very low frequency, VLF; low frequency, LF; medium high frequency, MHF; very high frequency, VHF).

well as a transformation type $\times$ word length interaction. In an attempt to prevent potential speed-accuracy tradeoffs from unduly affecting the present results, an analysis of covariance (ANCOVA) was conducted, with RT as the dependent variable and error rate in each condition as the covariate. The critical interactions of imaging ability $\times$ transformation type $\left[F(1,37)=3.88, M S_{\mathrm{e}}=\right.$ 538,927, $p=.056]$ and transformation type $\times$ length $\left[F(2,75)=46.63, M S_{\mathrm{e}}=61,495, p<.001\right]$ remained at least marginally reliable.

\section{Discussion}

An issue of central interest in the present investigation was whether reversed words would be analytically processed and nonreversed words would be holistically processed. Previous research has provided evidence that recognition of words consisting of a familiar spatialfrequency pattern is at least holistically biased (e.g., Allen \& Emerson, 1991; Allen \& Madden, 1990; Allen et al., in press; Healy, Conboy, \& Drewnowski, 1987; Healy \& Drewnowski, 1983; Healy, Oliver, \& McNamara, 1987; Johnson et al., 1989). However, there is little evidence that reversed words require analytic processing (although see Koriat \& Norman, 1985).

In the present study, RT and error analyses both revealed that mental transformation adversely affected performance. Furthermore, such transformation resulted in greater word-length effects. Indeed, an examination of Figure 2 shows that word-length effects for RT and errors are nonmonotonic for nonreversed words, but are monotonic for reversed words. These results are consistent with Koriat and Norman's (1985) prediction that mental transformation results in analytic processing, but that nonreversed objects (or objects that undergo minor transformation) tend to be holistically processed.

Another interest of the present study was whether imagery ability affects the processing resources of analytic processing. Experiment 1 provided some support for this notion, because the poor imagers showed greater mental transformation effects for RT than did the vivid imagers (i.e., a transformation type $\times$ imaging ability interaction). However, although transformation type interacted with word length, these two factors did not further interact with imaging ability. This three-way interaction would have provided more convincing evidence that individual differences in imaging ability affect analytic (letter-level) word recognition processes (e.g., Allen, Wallace, \& Waag, 1991), but the transformation type $\times$ imaging ability interaction did provide some evidence that is consistent with this view.

Finally, the interaction between transformation type and word frequency for RT and errors in Experiment 1 appears to be inconsistent with hybrid, holistically biased word recognition models such as the PISA. This is because the PISA model predicts that even words formed from initially letter-level codes will access the same mental lexicon as words recognized with a word-level code (Allen et al., 1992). An equally likely interpretation, however, is that the analytic mental transformation process that is hypothesized to occur before lexical access is more variable for reversed words than for nonreversed words because of the lower string familiarity of the reversed words. Indeed, the standard deviations for reversed words were larger than those for nonreversed 
words. Thus, the present interaction for mental transformation and word frequency is consistent with hybrid models of word recognition, if one assumes that a more variable encoding process for reversed words accounted for the transformation $\times$ word frequency interaction. ${ }^{2}$

\section{EXPERIMENT 2}

Experiment 2 was conducted to examine whether individual differences in imaging ability affect word processing when verbal ability is controlled for between these two types of imagers. All the words were reversed in the naming task used in Experiment 2, and presentation time was varied for a more sensitive manipulation of the utilization of analytic processing.

\section{Method}

Subjects. Forty Cleveland State University undergraduates participated in Experiment 2 for credit in an introductory psychology course. All of the subjects reported normal or correctedto-normal vision, and none had participated in Experiment 1 . The selection criteria for vivid and poor imagers were the same as those used for Experiment 1. When the WAIS-R Vocabulary Subscale scores and years-of-education data were analyzed as a function of imaging ability, there were no reliable differences for vocabulary scores $\left[F(1,38)=1.05, M S_{\mathrm{e}}=62.1, p>.30\right.$; vivid imagers' raw score mean $=62.5$, poor imagers' raw score mean $=60.0]$, or for years of education $\left[F(1,38)=0.61, M S_{\mathrm{e}}=1.03\right.$, $p>.44$; vivid imagers' mean years of education $=14.3$, poor $=$ 14.1].

Apparatus. The apparatus was the same as that used in Experiment 1.

Materials. The stimuli were the same as those used in Experiment 1 .

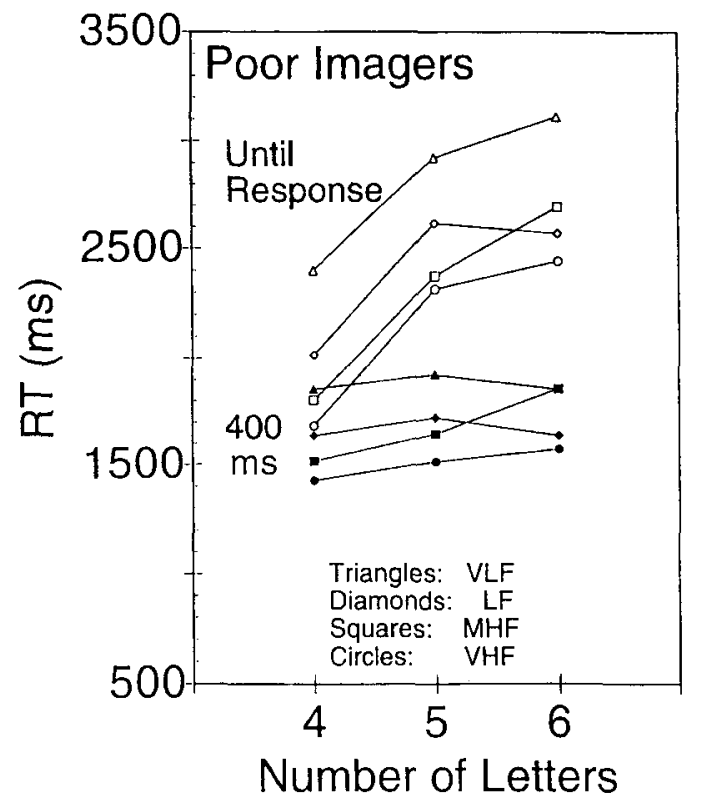

Procedure. The procedure in Experiment 2 was the same as that used in Experiment 1, except for the following exceptions. First, all the words were reversed, and the subjects were instructed to mentally transform them in order to pronounce them. Second, there were 432 experimental trials in Experiment 2 (Experiment 1 had 216 experimental trials), and two presentation-time blocks ( $400 \mathrm{msec}$ and presentation until response) of 216 trials each were used. Half of the subjects received the 400-msec block first, and the remaining half of the subjects received the presentation-untilresponse block first. Each word of the 432-word set of Allen et al. (1992) was used once. Word frequency and word length were randomly presented within each presentation-time trial block, so there were 18 trials in each presentation time $\times$ word frequency $x$ word length cell.

\section{Results}

Latency data. The RT data from Experiment 2 (see Figure 5) were analyzed with a 2 (imaging ability) $\times 2$ (presentation time: $400 \mathrm{msec}$ vs. presentation until response) $\times 4$ (word frequency) $\times 3$ (word length) ANOVA. Imaging ability was a between-subjects variable, but the remaining variables were repeated measures $(2 \times 2 \times 4 \times 3 \times 9=432$ experimental trials $)$. The results of the ANOVA indicated that the vivid imagers responded more rapidly $(1,726 \mathrm{msec})$ than the poor imagers $(2,046 \mathrm{msec})\left[F(1,38)=4.49, M S_{\mathrm{e}}=\right.$ $5,489,589, p<.05]$; the presentation-until-response duration resulted in longer RTs $(2,145 \mathrm{msec})$ than the 400 -msec presentation $(1,627 \mathrm{msec})[F(1,38)=59.66$, $\left.M S_{\mathrm{e}}=1,075,286, p<.001\right]$; the latencies increased as word frequency decreased $(\mathrm{VHF}=1,578 \mathrm{msec}, \mathrm{MHF}=$ $1,662 \mathrm{msec}, \mathrm{LF}=1,757 \mathrm{msec}, \mathrm{VLF}=1,906 \mathrm{msec}$ ) $\left[F(3,114)=72.72, M S_{\mathrm{e}}=104,991, p<.001\right]$; and that

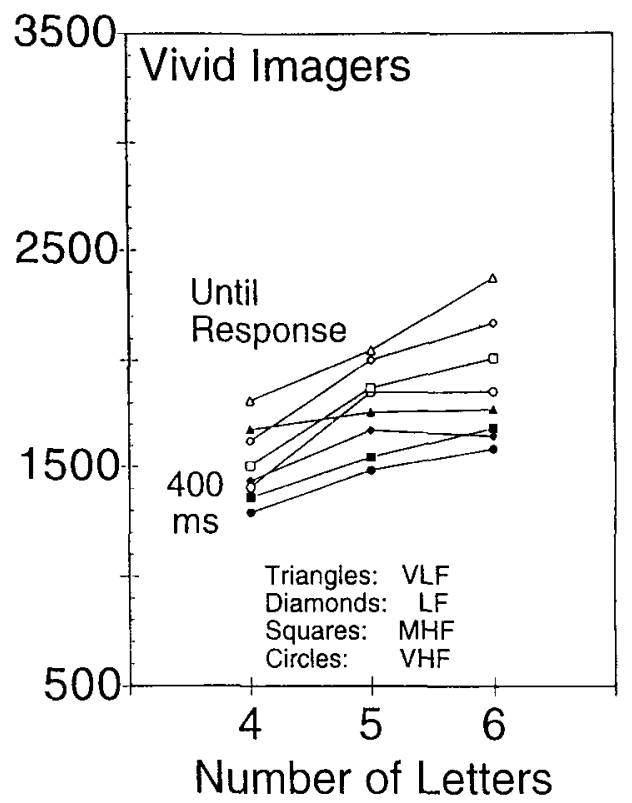

\footnotetext{
Figure 5. Vivid and poor imagers' reaction time (RT) data for Experiment 2 are illustrated as a function of presentation time (presentation until response vs. $400 \mathrm{msec}$ ), word length $(4,5$, or 6 letters), and word frequency (very low frequency, VLF; low frequency, LF; medium high frequency, MHF; very high frequency, VHF).
} 
latencies increased with increasing word length (four letters $=1,701 \mathrm{msec}$, five letters $=2,001 \mathrm{msec}$, six letters $=2,109 \mathrm{msec})\left[F(2,76)=109.49, M S_{\mathrm{e}}=126,420\right.$, $p<.001]$. Also, presentation time interacted with imaging ability $\left[F(1,38)=10.29, M S_{\mathrm{e}}=1,075,286, p<.01\right]$, and these two factors further interacted with word length $\left[F(2,76)=5.62, M S_{\mathrm{e}}=116,161, p<.05\right.$; i.e., a presentation time $\times$ imaging ability $\times$ word length interaction; see Figure 5]. A presentation time $\times$ word frequency interaction showed a relatively greater word frequency effect for the longer presentation duration $\left[F(3,114)=4.89, M S_{\mathrm{e}}=105,628, p<.01\right]$, and a word frequency $\times$ imaging ability interaction $[F(3,114)=$ $\left.4.11, M S_{\mathrm{e}}=104,991, p<.01\right]$ revealed that the poor imagers had a greater word frequency advantage $(519 \mathrm{msec})$ than did the vivid imagers $(328 \mathrm{msec})$ (see Figure 5).

When the data from the subjects were analyzed separately by imaging ability, the vivid and poor imagers both showed main effects for presentation time, word frequency, and word length, as well as a presentation time $\times$ word length interaction. Also, when the simple main effects for presentation time were examined across imaging ability, the data revealed that the poor imagers took longer to name reversed words in the presentationuntil-response condition $\left[F(1,38)=7.83, M S_{\mathrm{e}}=\right.$ $365,841, p<.01]$, but they did not take significantly longer to name reversed words in the $400-\mathrm{msec}$ condition $[F(1,38)<1.00]$.

Also, the critical imaging ability $\times$ presentation time $\times$ word length interaction was ambiguous, because the poor imagers actually showed a smaller word-length disadvantage than did the vivid imagers for the $400-\mathrm{msec}$ presentation condition, but the poor imagers showed a larger word-length disadvantage than did the vivid imagers for the presentation-until-response condition (Figure 5). To address this issue in further detail, we analyzed the presentation-until-response data separately to determine whether an imagery ability $\times$ word length interaction was present; it was $\left[F(2,76)=4.26, M S_{\mathrm{e}}=\right.$ $149,740, p<.05]$. When the $400-\mathrm{msec}$ presentation data were analyzed separately, the imaging ability $\times$ word length interaction was no longer reliable $[F(2,76)=$ 1.34].

Error data. The mean error data for Experiment 2 are presented in Figure 6. The analysis conducted on the error data from Experiment 2 revealed that the poor imagers committed more errors (mean error $=36.4 \%$; note that chance performance for a naming task is not $50 \%$ ) than did the vivid imagers $(23.7 \%)[F(1,38)=7.21$, $\left.M S_{\mathrm{e}}=175.12, p<.05\right]$; the subjects committed more errors on 400 -msec trials $(41.0 \%)$ than on presentationuntil-response trials $(19.0 \%)\left[F(1,38)=128.04, M S_{\mathrm{e}}=\right.$ $5.75, p<.001]$; the subjects showed a word-length disadvantage (four letters $=18.3 \%$, five letters $=31.5 \%$, six letters $=40.2 \%)\left[F(2,76)=218.69, M S_{\mathrm{e}}=5.75\right.$, $p<.001]$; and that the subjects showed a word frequency effect $(\mathrm{VHF}=24.5 \%, \mathrm{MHF}=25.2 \%, \mathrm{LF}=$ $28.6 \%, \mathrm{VLF}=41.4 \%)\left[F(3,114)=123.74, M S_{\mathrm{e}}=\right.$ $3.87, p<.001]$. Decreased presentation time exacerbated word-length effects $\left[F(2,76)=33.54, M S_{\mathrm{e}}=\right.$ $5.78, p<.001]$ and word frequency effects $[F(3,114)=$ $\left.3.34, M S_{\mathrm{e}}=3.94, p<.05\right]$. Also, the poor imagers showed greater word frequency effects for errors than did the vivid imagers $\left[F(3,114)=7.04, M S_{\mathrm{e}}=3.87\right.$, $p<.01]$. Finally, when the Experiment 2 error data were
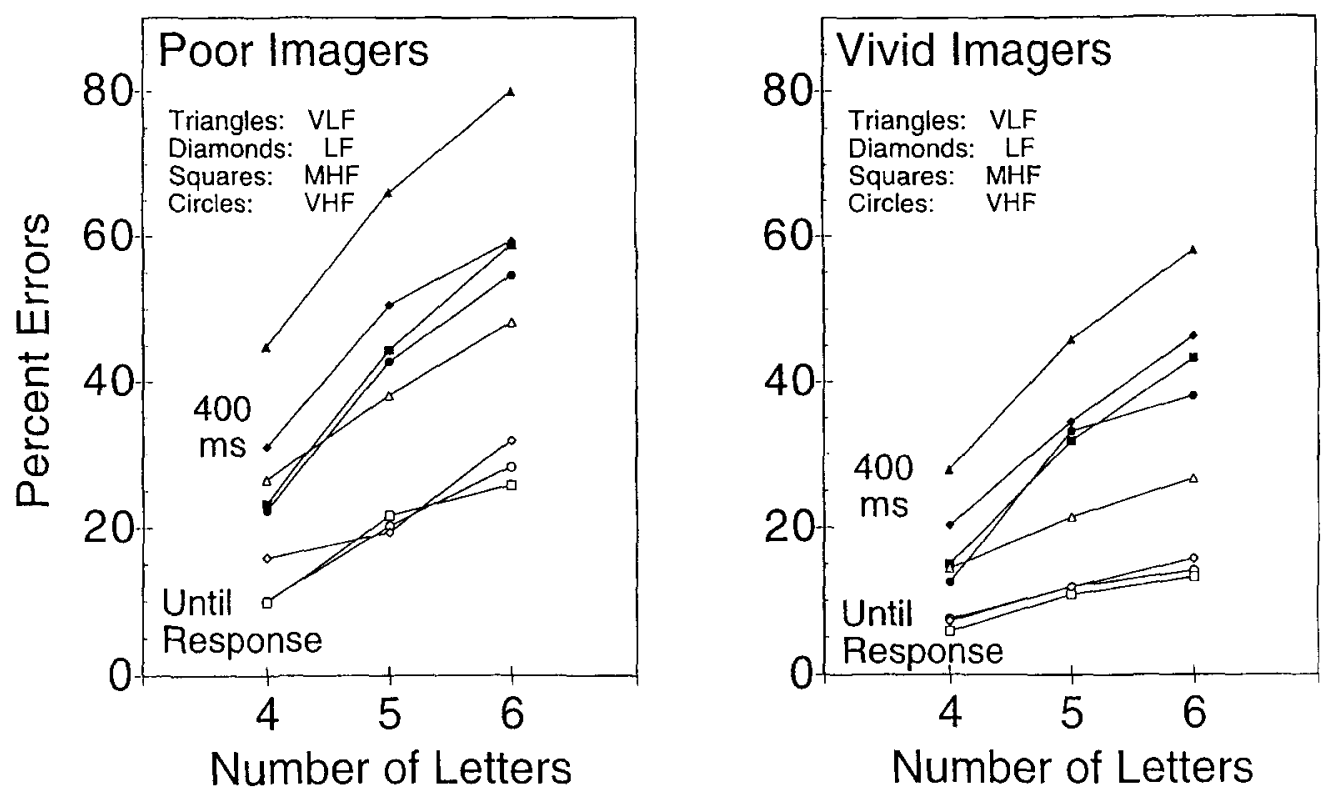

Figure 6. Vivid and poor imagers' mean percent error data for Experiment 2 are illustrated as a function of presentation time (presentation until response vs. $400 \mathrm{msec}$ ), word length $(4,5$, or 6 letters), and word frequency (very low frequency, VLF; low frequency, LF; medium high frequency, MHF; very high frequency, VHF). 
analyzed separately by imaging ability, the poor and vivid imagers both revealed main effects for presentation time, word length, and word frequency, as well as presentation time $\times$ word length interactions.

As was done for Experiment 1, we attempted to rule out speed-accuracy confounds by analyzing the Experiment 2 data with an ANCOVA (dependent variable = RT; covariate $=$ errors). However, the significance of RT interactions was unchanged when the effect of errors was extracted. The results suggest that a speed-accuracy tradeoff did not confound the present results.

\section{Discussion}

The vivid and poor imagers who participated in Experiment 2 did not differ significantly with regard to verbal ability (as indexed by WAIS-R vocabulary performance) or years of education. The poor imagers, however, required a significantly longer period of time to pronounce reversed words than did the vivid imagers, and this effect was exacerbated when task difficulty was increased by increasing word length and presentation time. Assuming that transforming a word tends to result in analytic processing (e.g., Koriat \& Norman, 1985), these results provide evidence that imaging-ability differences in processing resources are related to differences in the utilization of analytic processes. Specifically, these data indicate that poor imagers evidence fewer analytic processing resources than do vivid imagers in a naming task in which they are required to mentally reverse words in order to name them.

Finally, in Experiment 2, word frequency interacted with imaging ability, although this interaction did not occur for Experiment 1. We attribute these results to the fact that most of the processing in Experiment 2 involved primarily analytic processes (i.e., all the words were reversed), whereas only half of the Experiment 1 trials involved primarily analytic processes (i.e., half of the words were reversed). Consequently, the poor imagers may have evidenced greater word frequency effects in Experiment 2 than did the vivid imagers because they had fewer resources with which to conduct analytic processing.

\section{GENERAL DISCUSSION}

The present investigation provides support for Koriat and Norman's (1985) and Allen et al.'s (in press) contention that subjects tend to use holistic processing for words with a familiar spatial-frequency pattern, but tend to use analytic processing for transformed words with an unfamiliar pattern. The strongest evidence for this contention was found in Experiment 1 . The subjects revealed a word-length disadvantage for reversed words, but not for nonreversed words (see Figure 3).

With regard to imaging, we found that the appropriate issue is not simply whether imagery consists of holistic or analytic factors. An additional issue of considerable importance is: Under what conditions do indi- viduals use a given imaging process? In the present Experiment 1 , there were no appreciable differences in RT performance between the poor and vivid imagers when words were presented in a nonreversed manner (e.g., JUMP). However, when the subjects were required to transform reversed words (e.g., PMUJ to JUMP), the poor imagers took significantly longer to respond than the vivid imagers (see Figure 3 ). Thus, the present naming data indicate that poor imagers are less efficient at utilizing analytic processing during visual word recognition than are vivid imagers - even though poor imagers tend to favor an analytic cognitive style (Crawford \& Allen, 1983). This interpretation is based upon the assumption that analytic (letter-level) processing during word recognition involves more and smaller pieces of information than does holistic (word-level) processing (Allen \& Madden, 1990; Allen, Madden, \& Crozier, 1991). Also, poor imagers appear to have fewer processing resources than do vivid imagers, due to higher baseline internal noise levels (Allen, Wallace, \& Waag, 1991). The RT results from Experiment 2, which showed that presentation time and word length interacted with imaging ability even when verbal ability was controlled for, are consistent with this interpretation if one assumes that longer presentation times result in suboptimal sensory-response activation.

In summary, the present investigation provides further evidence that poor imagers perform more slowly and/or less efficiently than vivid imagers on a cognitive task. Even though, in general, poor imagers tend to favor an analytic processing style (Crawford \& Allen, 1983; Wallace, 1990), and vivid imagers tend to favor a holistic processing style (Wallace, 1991b), task demands in the present investigation appear to exceed resource limitations on the ability of poor imagers to utilize an analytic processing strategy.

\section{REFERENCES}

Allen, P. A., \& Emerson, P. E. (1991). Holism revisited: Evidence for parallel independent word-level and letter-level processors during word recognition. Journal of Experimental Psychology: Human Perception \& Performance, 17, 489-511.

Allen, P. A., \& MAdDEN, D. J. (1990). Evidence for a parallel input serial analysis model of word processing. Journal of Experimental Psychology: Human Perception \& Performance, 16, 48-64.

Allenn, P. A., Madden, D. J., \& Crozier, L. C. (1991). Adult age differences in letter-level and word-level processing. Psychology \& Aging, 6, 261-271.

Allen, P. A., MCNEAL, M., \& KvaK, D. (1992). Perhaps the lexicon is coded as a function of word frequency. Journal of Memory \& Language, 31, 826-844.

Allen, P. A., Wallace, B., \& WaAg, E. (1991). Effect of imagery ability on letter-level and word-level processing. Perception \& Psychophysics, 49, 295-300.

Allen, P. A., Wallace, B., \& Weber, T. A. (in press). Influence of case type, word frequency, and exposure duration on visual word recognition. Journal of Experimental Psychology: Human Perception \& Performance.

Chara, P. J. (1989). A questionable questionnaire: A rejoinder to Marks. Perceptual \& Motor Skills, 68, 159-162.

Crawford, H. J., \& Allen, S. N. (1983). Enhanced visual memory during hypnosis as mediated by hypnotic responsiveness and cog- 
nitive strategies. Journal of Experimental Psychology: General, 112. 662-685.

FonOR, J. A. (1983). Modularity of mind. Cambridge, MA: Bradford.

GorDON, R. (1949). An investigation into some of the factors that favour the formation of stereotyped images. British Journal of Psychology, 39, 156-167.

Healy, A. F., Conboy, G. L., \& Drewnowski, A. (1987). Detecting letters in continuous text: Effects of display size. In B. Britton \& S. Glynn (Eds.), Executive control processes in reading (pp. 279296). Hillsdale, NJ: Erlbaum.

HEALY, A. F., \& DREWNOSKI, A. (1983). Investigating the boundaries of reading units: Letter detection in misspelled words. Journal of Experimental Psychology: Human Perception \& Performance, 9, 413-426.

Healy, A. F., Oliver, W. L., \& McNamara, T. P. (1987). Detecting letters in continuous text: Effects of display size. Journal of Experimental Psychology: Human Perception \& Performance, 13, 279290.

Johnson, N. F., Allen, P. A., \& Strand, T. L. (1989). On the role of word frequency in the detection of component letters. Memory \& Cognition, 17, 474-482.

Koriat, A., \& NoRman, J. (1985). Reading rotated words. Journal of Experimental Psychology: Human Perception \& Performance, 11, 490-508

KORIAT, A., \& NORMAN, J. (1989). Why is word recognition impaired by disorientation while the identification of single letters is not? Journal of Experimental Psychology: Human Perception \& Performance, 15, 153-163.

KrUeger, L. E. (1979). Features versus redundancy: Comments on Massaro, Venezky, and Taylor's "Orthographic regularity, positional frequency, and visual processing of letter strings." Journal of Experimental Psychology: General, 108, 125-130.

Krueger, L. E. (1989). Detection of intraword and interword letter repetition: A test of the word unitization hypothesis. Memory \& Cognition, 17, 48-57.

KučEra, H., \& Francis, W. N. (1967). Computational analysis of present-day American English. Providence, RI: Brown University Press.

MARKS, D. F. (1973). Visual imagery differences in the recall of pictures. British Journal of Psychology, 64, 17-24.

Massaro, D. W., Venezky, R. L., \& Taylor, G. A. (1979). Orthographic regularity, positional frequency, and visual processing of letter strings. Journal of Experimental Psychology: General, 108, 107-124.

NAvon, D. (1984). Resources-A theoretical soup stone? Psychological Review, 91, 216-234.

SCHNEIDER, W. (1988). Micro Experimental Laboratory: An integrated system for IBM PC compatibles. Behavior Research Methods, Instruments, \& Computers, 20, 206-217.

STREET, R. F. (1931), A gestalt completion test (Contributions to Education No. 481). New York: Teachers College Press, Columbia University .

Thurstone, L. L., \& Jeffrey, T. E. (1966). Closure Speed Test administration manual. Chicago: Industrial Relations Center, University of Chicago.

WALLACE, B. (1988). Imaging ability, visual search strategies, and the unvividness paradox. Journal of Mental Imagery, 12, 173-184.

WALLACE, B. (1990). Imagery vividness, hypnotic susceptibility, and the perception of fragmented stimuli. Journal of Personality \& Social Psychology, 58, 354-359.

WALLACE, B. (1991a). Hypnotic susceptibility, imaging ability, and information processing: An integrative look. In R. G. Kunzendorf (Ed.), Mental imagery (pp. 89-100). New York: Plenum.

WALLACE, B. (1991b). Imaging ability and performance in a proofreading task. Journal of Mental Imagery, 15, 177-188.

\section{NOTES}

1. For the purposes of this paper, a processing resource is defined as "any internal input essential for processing (e.g., locations in storage, communication channels) that is available in quantities that are limited at any point in time" (Navon, 1984, p. 217). Although Navon noted that the concept of processing resources is not without problem, he concluded that it probably is a useful heuristic for examining resource pools.

2. One potential account of the larger word frequency advantage for reversed words than for nonreversed words is that reversed lower frequency words have fewer orthographic cues (Massaro, Venezky, \& Taylor, 1979) or less sequential redundancy (Krueger, 1979) than reversed higher frequency words or higher frequency and lower frequency nonreversed words. However, it would seem that longer words would provide more orthographic and sequential redundancy information than would shorter words, but the word frequency advantage for longer words was not smaller than that for shorter words (see Figure 3). Furthermore, it took the subjects longer to name the reversed six-letter words than the reversed four-letter words. It would seem that the greater orthographic and sequential redundancy information contained in longer words should result in the opposite effect if these factors were responsible for the present transformation type $\times$ word frequency interaction.

(Manuscript received September 7, 1993; revision accepted for publication October 20, 1993.) 